\title{
The Implications of Second-Order Functional Derivatives on Error Estimation in Adaptive Finite Element Analysis for Electromagnetics
}

\author{
Steve McFee and Dennis Giannacopoulos \\ Department of Electrical \& Computer Engineering, McGill University, Canada
}

\begin{abstract}
The potential advantages and related costs of using second-order functional mesh discretization derivatives for error estimation in adaptive finite element analysis (FEA) for electromagnetics are investigated. Second-order indicators are proposed to identify and stabilize erroneous first-order error distributions that arise in unbalanced discretization regions. Effective combined derivative estimators are introduced and evaluated in practical applications.
\end{abstract}

Index Terms- Electromagnetic analysis, finite element methods, adaptive systems, error analysis.

\section{INTRODUCTION}

The study of error estimation for finite element adaption in electromagnetics has been the focus of a great amount of work over the past ten years, and now represents a well-established research area [1], [2]. Today, a variety of error indicators are used, and many of the most effective ones are based on local derivatives of the approximated fields [3], [4]. Common examples include field discontinuity, PDE residual, local energy and functional gradient measures. As with all local error estimation methods, derivative-based approaches can yield misleading results when used with insufficient or unbalanced discretizations [5], [6]. It is not uncommon for evolving, unconverged finite element models to give rise to locally smooth regions of high relative error, which yield wellbehaved first-order derivatives. The difficulty with error estimation is that well-behaved first-order derivatives are primarily correlated with indicators of stability and low error. The purpose of this contribution is to introduce and investigate the use of second-order functional derivative indicators, which are largely unaffected by such problematic error distributions.

\section{SECond-order functional Derivative INDICATORS}

Functional gradient error indicators associated with optimal discretization based refinement criteria have been employed successfully in adaptive finite element methods for electromagnetics [4]. Despite their demonstrated effectiveness, these first-order functional derivative based indicators are not immune to the problems associated with guiding adaptive methods reliably and efficiently when used with insufficient or unbalanced discretizations. Under such conditions, ineffective discretizations may evolve

Manuscript received June 1, 1998. This work was supported in part by the Natural Sciences and Engineering Research Council of Canada. during the course of the adaption. Consequently, poor adaption performance results may be observed over part, or throughout the entire adaptive process, if problematic error distributions due to unstable first-order functional derivative error indicators are not detected and corrected.

Second-order functional derivatives can be used to analyze the stability and estimate the reliability of first-order derivative-based local error assessments. In practice, locally smooth regions of high relative error in finite element models are usually unstable, and easy to detect with second-order derivative tests. Electromagnetic systems that possess translational or rotational symmetries may be analyzed using 2-D finite element formulations, and second-order functional derivative based error indicators are defined in terms of derivatives with respect to element vertex positions for such 2-D systems. For example, in Cartesian problems where the field solution variation is independent of the coordinate variable $z$, i.e., $u=u(x, y)$, the second-order functional derivatives may be computed directly. Consider a scalar triangular element with vertex positions $\left(x_{l}, y_{l}\right), l=1,2,3$. For Helmholtz systems, the $x$ - and $y$-components of the second-order functional derivatives may be readily determined from the matrix forms:

$$
\frac{1}{2} \mathbf{u}^{T} \mathbf{P} \mathbf{u}, \text { and } \frac{1}{2} \mathbf{u}^{T} \mathbf{Q} \mathbf{u},
$$

respectively, evaluated over the elements that share the vertex in question. Here, $\mathbf{u}$ is the field solution vector. The square matrices $\mathbf{P}$ and $\mathbf{Q}$ contain the $x$ and $y$ second-order derivative information, respectively, that corresponds to the Laplacian part of the functional for vertex $l(l=1,2,3)$ of the triangular element. The entries of the matrices $\mathbf{P}$ and $\mathbf{Q}$ are defined by:

$$
\begin{aligned}
P_{i j}= & \frac{1}{4 A^{2}} \sum_{m=1}^{3} \sum_{n=1}^{3}\left[c_{l} \frac{\partial\left(c_{m} c_{n}\right)}{\partial x_{l}}-b_{l} \frac{\partial\left(b_{m} b_{n}\right)}{\partial y_{l}}\right. \\
& \left.+2 A \frac{\partial^{2}\left(c_{m} c_{n}\right)}{\partial x_{l}^{2}}\right] I_{i j m n}-\frac{1}{A}\left(b_{l}+c_{l}\right) V_{i j},
\end{aligned}
$$

and

$$
\begin{aligned}
Q_{i j}= & \frac{1}{4 A^{2}} \sum_{m=1}^{3} \sum_{n=1}^{3}\left[b_{l} \frac{\partial\left(b_{m} b_{n}\right)}{\partial y_{l}}-c_{l} \frac{\partial\left(c_{m} c_{n}\right)}{\partial x_{l}}\right. \\
& \left.+2 A \frac{\partial^{2}\left(b_{m} b_{n}\right)}{\partial y_{l}^{2}}\right] I_{i j m n}-\frac{1}{A}\left(b_{l}+c_{l}\right) W_{i j}
\end{aligned}
$$

where $A$ is the element area; and $b_{i}$ and $c_{i}$ are geometric parameters related to the element's vertex positions, which can be defined as follows with the subscripts progressing modulo 3 :

$$
\begin{aligned}
b_{i} & =y_{i+1}-y_{i-1}, \text { and } \\
c_{i} & =x_{i-1}-x_{i+1} .
\end{aligned}
$$


$I_{i j m n}$ is the elemental integral (in homogeneous coordinates) of the product of the derivatives of the $i$ th and $j$ th basis functions, with respect to the $m$ th and $n$th simplex coordinates. It should be noted that the "mixed". second-order functional derivative terms with respect to both the $2 x$ and $y$ element vertex positions are incorpes rated into th tefinitions of matrices $\mathbf{P}$ and $\mathbf{Q}$ in (2)

(3) above. Furthermore, $V_{i j}$ and $W_{i j}$ are directly related to the first-order functional derivatives, with respect to element vertex positions, as defined in [4]:

$$
\frac{1}{2} \mathbf{u}^{T} \mathbf{V u}-b_{l} \frac{k^{2}}{2} \mathbf{u}^{T} \mathbf{B u}+b_{l} \mathbf{u}^{T} \mathbf{B f}
$$

and

$$
\frac{1}{2} \mathbf{u}^{T} \mathbf{W} \mathbf{u}-c_{l} \frac{k^{2}}{2} \mathbf{u}^{T} \mathbf{B u}+c_{l} \mathbf{u}^{T} \mathbf{B f}
$$

Here, $\mathbf{u}$ and $\mathbf{f}$ are the field solution and the source term vectors, respectively, and $k$ is the free-space wave number of the system. The square matrices $\mathbf{V}$ and $\mathbf{W}$ contain the $x$ and $y$ first-order derivative information, respectively, that corresponds to the Laplacian part of the functional for vertex $l(l=1,2,3)$ of a triangular element. The entries of the matrices $\mathbf{V}$ and $\mathbf{W}$ are defined by:

$V_{i j}=\frac{1}{4 A^{2}} \sum_{m=1}^{3} \sum_{n=1}^{3}\left[2 A \frac{\partial\left(c_{m} c_{n}\right)}{\partial x_{l}}-\left(b_{m} b_{n}+c_{m} c_{n}\right) b_{l}\right] I_{i j m n}$,

and

$W_{i j}=\frac{1}{4 A^{2}} \sum_{m=1}^{3} \sum_{n=1}^{3}\left[2 A \frac{\partial\left(b_{m} b_{n}\right)}{\partial y_{l}}-\left(b_{m} b_{n}+c_{m} c_{n}\right) c_{l}\right] I_{i j m n}$.

The $x$ and $y$ first-derivatives of the wave and source functional terms are given by the second and third terms in each of (8) and (9), respectively, where $B_{i j}$ is the elemental integral (in homogeneous coordinates) of the product of the $i$ th and $j$ th basis functions. It may be noted that the partial derivatives of $\left(b_{m} b_{n}\right)$ and $\left(c_{m} c_{n}\right)$ with respect to the element vertex positions, which appear in (8) and (9), can be determined directly from (4) and (5) and are given for reference in Table I. Subsequently, the corresponding second-order partial derivatives of these product terms, which appear in (2) and (3), may be readily determined from (4), (5), and Table I. It has been previously shown that first-order functional derivative quantities are closely related to the variational principle used to determine the solution to the finite element problem [4], and they are efficient to compute. Similarly, the new secondorder quantities are inexpensive to compute since the only extra terms required are numerical constarits which can be tabulated once and for all.

As in the first-derivative case [4], the second-order functional derivative formulas derived above are valid for any choice of legitimate finite element basis functions. Furthermore, the functional derivatives may be computed for uniform- or mixed-order meshes as may be required by specific refinement models such as $h-, p$-, or $h p$-adaptive methods. Although the above formulation has been derived for scalar Helmholtz systems, it is interesting to note that the second-order derivatives of the wave and source terms of the functional, with respect to the vertex positions, are zero. This suggests that 2-D Laplace systems
TABLE I

EXPLICIT FORMS OF $\partial\left(b_{m} b_{n}\right) / \partial y_{l}$ IN TERMS OF $b_{i}$

\begin{tabular}{cccc}
\hline$(m, n) / l$ & 1 & 2 & 3 \\
\hline$(1,1)$ & 0 & $2 b_{1}$ & $-2 b_{1}$ \\
$(1,2),(2,1)$ & $-b_{1}$ & $b_{2}$ & $b_{1}-b_{2}$ \\
$(2,2)$ & $-2 b_{2}$ & 0 & $2 b_{2}$ \\
$(1,3),(3,1)$ & $b_{1}$ & $b_{3}-b_{1}$ & $-b_{3}$ \\
$(2,3),(3,2)$ & $b_{2}-b_{3}$ & $-b_{2}$ & $b_{3}$ \\
$(3,3)$ & $2 b_{3}$ & $-2 b_{3}$ & 0 \\
\hline
\end{tabular}

Note: explicit forms of $\partial\left(c_{m} c_{n}\right) / \partial x_{l}$ in terms of $c_{i}$ may be obtained by replacing $b_{i}$ with $-c_{i}$.

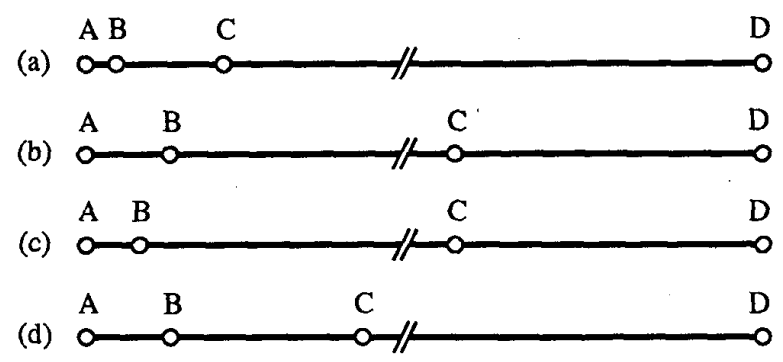

Fig. 1. First-order, three element, discretization for 1-D electrostatic potential analysis of point charge benchmark: (a) globally optimal mesh; (b) unbalanced mesh; (c) mesh (b) after local optimization of position of node B; (d) mesh (b) after local optimization of position of node $C$. Note: the meshes are radial; the point charge is located at the origin; node $\mathrm{A}$ is fixed at $r=0.1$; node $\mathrm{D}$ is fixed at $r=10$; and the various positions of nodes $B$ and $C$ are specified in Table II.

may benefit most from error estimation based on using both first- and second-order functional derivatives.

\section{RESULtS}

Three benchmark systems are presented to illustrate the error estimation pitfalls that can occur with insufficient or unbalanced discretizations, and the potential value of using second-order derivative methods to avoid them. Specifically, a simple 1-D free-space example, a 2-D Laplace system, and a 2-D Helmholtz system were examined in order to investigate the practical significance of the new approach.

\section{A. Free-space point charge test system}

The analysis details and results for the 1-D example, based on resolving the classical point charge benchmark, are given in Fig. 1 and Table II. To focus ideas, a functional gradient indicator was used to estimate nodal errors, and locally optimal $r$-refinements were used to update the discretization. Under these conditions, small first-derivatives indicate high accuracy; and high second-derivatives indicate instability and unreliable first-derivatives. Finally, the standard 'Taylor's expansion provides a simple way of combining the two derivatives into a single indicator. Note: the first-derivative erroneously indicates that refining $\mathrm{B}$ would be best; the second-derivative suggests that the first-derivative result is unreliable; the combined indicator correctly selects $\mathrm{C}$ as best for refinement. 
TABLE II

Numerical Results for Point Charge Benchmark

\begin{tabular}{ccccc}
\hline Mesh & (a) & (b) & (c) & (d) \\
\hline B Pos. & 0.2028 & 0.3757 & 0.2723 & 0.3757 \\
$d F / d B$ & 0 & 17.51 & 0 & 20.84 \\
$d^{2} F / d B^{2}$ & 235.2 & 0.1425 & 0.1143 & 101.52 \\
B Com. & 117.6 & 17.58 & 0.0572 & 71.6 \\
C Pos. & 0.5485 & 9.0 & 9.0 & 0.9946 \\
$d F / d C$ & 0 & 0.192 & 0.1784 & 0 \\
$d^{2} F / d C^{2}$ & 0.043 & 67.46 & 63.38 & 0.0581 \\
C Com. & 0.0215 & 33.92 & 31.87 & 0.0291 \\
F Error & $44 \%$ & $111 \%$ & $99 \%$ & $69 \%$ \\
\hline
\end{tabular}

These tabulated results correspond to the 1-D meshes described in Fig.1. B Pos. and C Pos. indicate the positions of nodes $B$ and $C$, respectively. $d F / d B$ and $d^{2} F / d B^{2}$ indicate the first- and second-derivatives of the functional with respect to a positional displacement of node $\mathrm{B}$ in the positive radial direction. $d F / d C$ and $d^{2} F / d C^{2}$ indicate similar derivatives w.r.t. node C. B Com. and $\mathrm{C}$ Com. refer to the combined first- and second-order positional derivative results corresponding to nodes $B$ and $C$, respectively. $F$ Error indicates the error in the functional value.

\section{B. 2-D Laplace test system}

The Laplace benchmark system is described by Fig. 2 . It is one-quarter of a square coaxial line in cross-section - the standard "L" problem. The conductor boundary conditions are $1 \mathrm{~V}$ and $0 \mathrm{~V}$ as indicated; and the symmetry planes are labeled $N$.

Performance results for second-order $h$-adaption studies on functional convergence are presented in Fig.3. The uniform $h$-refinement baseline (A) is included for comparison with $h$-refinement based on a first-order derivative error estimator (B) and a combined first- and secondorder error estimator (C). A $50 \%$ increment in the number of degrees of freedom (DOF) per adaptive step was used to update the discretizations for these studies, excluding the uniform refinement procedures. For the specific type of error estimator examined here (Type- $A$ [4]), and for the given amount of DOF update used per adaptive step, these results demonstrate a marginal improvement in performance for functional accuracy levels between $1 \%$ and $0.1 \%$ when the combined error estimator is used (C) versus the first-derivative estimator (B), and a more significant improvement for functional accuracy levels beyond $0.1 \%$.

The performance results for second-order $h$-adaption studies based on a second type of error estimator (Type- $B$ [4]) are presented in Fig.4. In this case, a $20 \%$ increment in the number of DOF per adaptive step was used. These results clearly demonstrate the practical value of the new approach and support the hypothesis that second-order functional derivatives can be used to analyze the stability and estimate the reliability of first-order derivative based local error assessments: curve (A) shows the uniform $h$ refinement baseline for comparison; curves (B) and (C) show the relative $h$-adaption performance for the firstorder and the combined first- and second-order based error estimation methods, respectively. Two example $h$ refined meshes corresponding to curve (B) and curve (C) are presented in Fig.5 to further illustrate the potential benefits of using the combined-derivative approach.

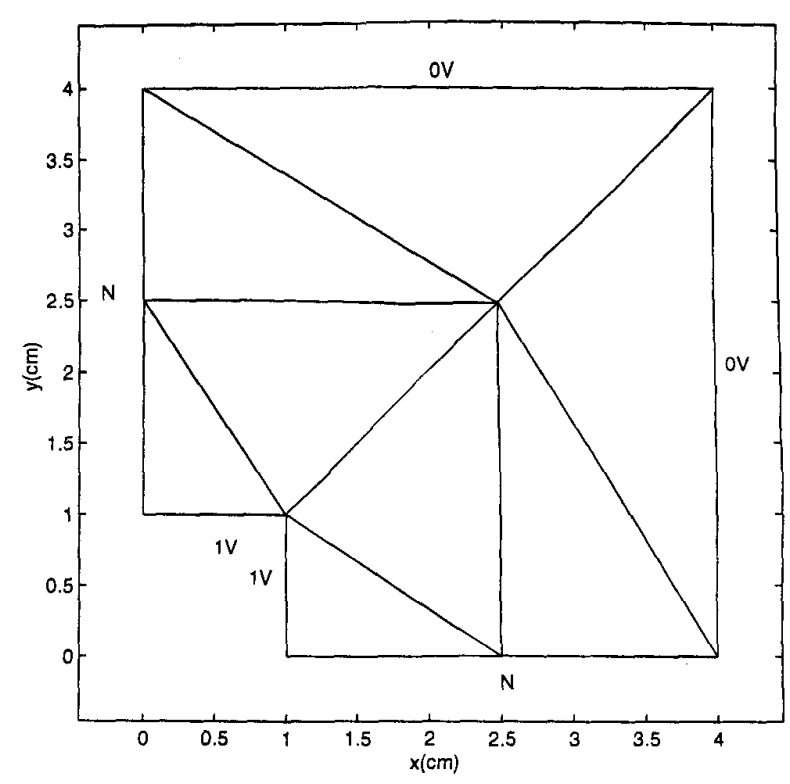

Fig. 2. Laplace benchmark system; initial $h$-mesh ( 8 triangles).

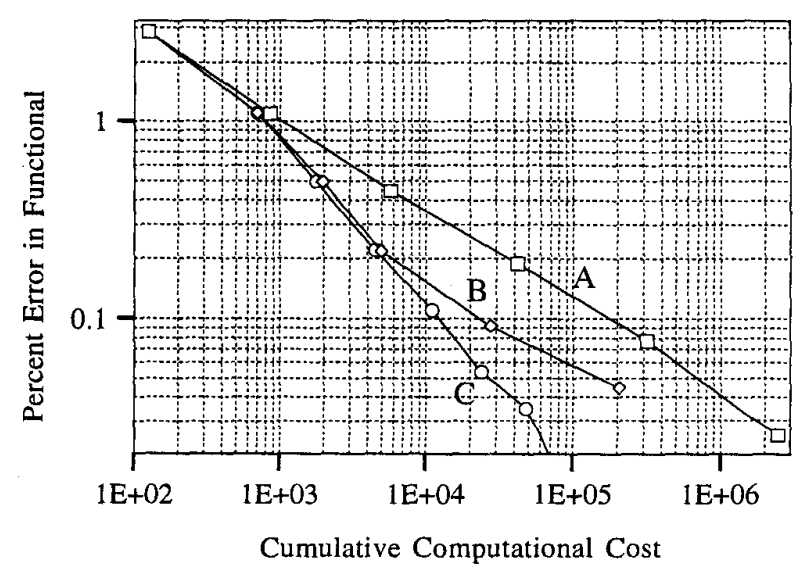

Fig. 3. Cumulative cost of adaption versus percent error in functional.

\section{2-D Helmholtz test system}

The Helmholtz benchmark system is described by Fig.6. It is an octagonal microstrip patch of size $d(34 \mathrm{~mm})$, where $\lambda=0.616 d$ ( $\lambda$ is the wavelength in the dielectric substrate below the patch). The device has only one port, at the end of the microstrip transmission line connected to the left hand side of the patch. In this study, the boundaries have been modeled as perfect magnetic walls to yield a two-dimensional electric field system. The objective for this benchmark is to find the phase angle of the reflection coefficient at the input port $P$.

Performance results for $p$-adaption studies on phase angle convergence are reported in Table III. The uniform p-refinement baseline result is presented for comparison. The $p$-discretizations ranged from orders 1 through 10 , and a $20 \%$ increment in the number of DOF per adaptive step was used to improve the discretizations for the methods considered, excluding the uniform refinement proce- 


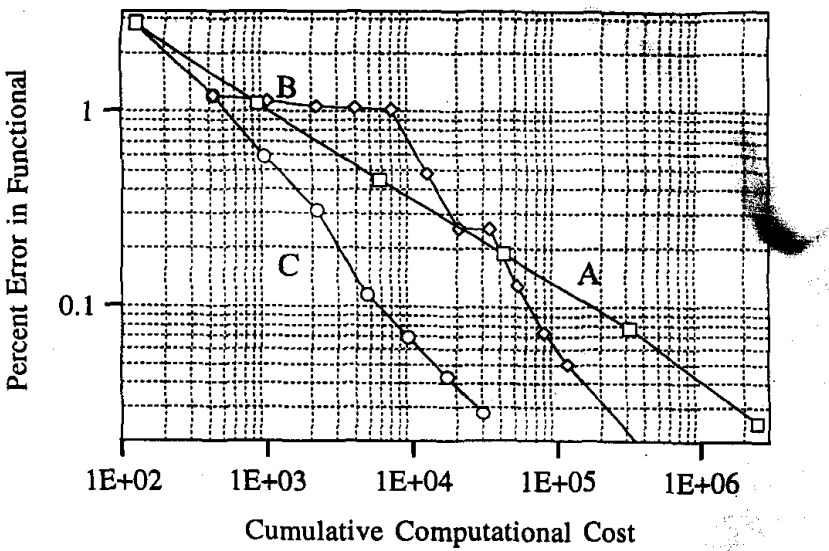

Fig. 4. Cumulative cost of adaption versus percent error th functional.

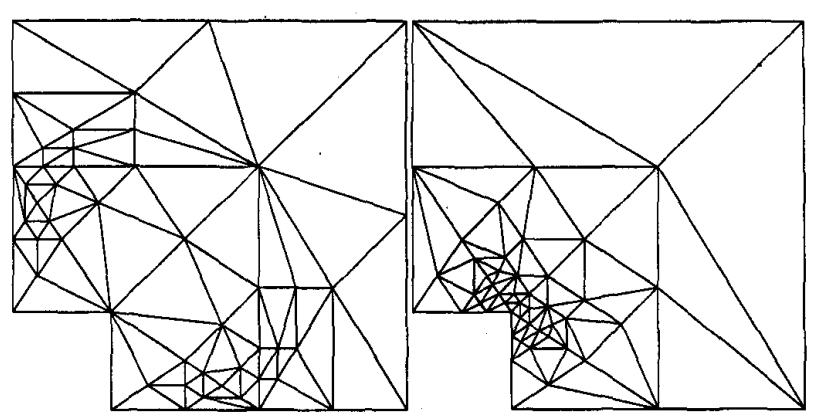

Fig. 5. Example of an ineffective $h$-refinement discretization due to unstable first-order derivative-based error estimator (left); Example of an effective $h$-refinement discretization due to combined first- and second-order derivative-base error estimator (right); 84 elements in each mesh.

dures. For the type of error estimator investigated (Type$B$ [4]), an average savings of approximately $20 \%$ in the number of DOF required to achieve phase error levels between $5.0^{\circ}$ and $0.5^{\circ}$ was observed for the combinedderivative approach, relative to the first-order derivative method.

It should be noted that the selected results presented in this section comprise a representative sampling of the full findings obtained over the course of the complete investigation.

\section{Conclusions}

New error estimators, based on combined first- and second-order functional derivatives for scalar 2-D Poisson and Helmholtz FEA, have been introduced and evaluated for adaption. The performance results for the benchmark systems investigated demonstrate that second-order derivative indicators can identify and stabilize erroneous first-order error distributions, and that combined derivative error estimation methods can be successfully used in adaptive finite element solvers to more reliably and economically distribute DOF over a problem domain.

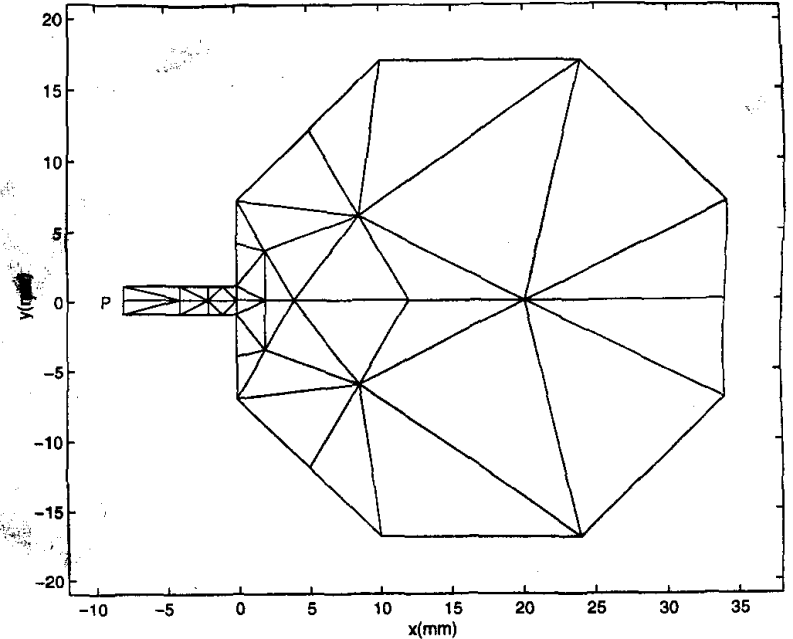

Fig. 6. Helmholtz benchmark system; initial $p$-mesh (44 triangles).

TABLE III

Discretization Level versus Phase Error in Degrees

\begin{tabular}{ccccccc}
\hline Method / \#DOF & $5.0^{\circ}$ & $2.5^{\circ}$ & $1.25^{\circ}$ & $1.0^{\circ}$ & $0.75^{\circ}$ & $0.5^{\circ}$ \\
\hline Uniform & 360 & 385 & 440 & 490 & 530 & 580 \\
First-order & 320 & 390 & 450 & 490 & 540 & 600 \\
Combined & 265 & 322 & 400 & 420 & 435 & 450 \\
\hline
\end{tabular}

This data corresponds to the $p$-adaption results for the 2-D mesh described in Fig.6. Uniform indicates uniform p-adaption. First-order indicates first-order functional derivative based error indicators were used to guide the $p$-adaption. Combined indicates both first- and second-order functional derivative based error indicators were used.

\section{REFERENCES}

[1] P. Fernandes, P. Girdinio, P. Molfino and M. Repetto, "Local error estimates for adaptive mesh refinement," IEEE Trans. Magn., vol. 24(1), pp. 299-302, 1988.

[2] D. Giannacopoulos and S. McFee, "Towards optimal $h-p$ adaption near singularities in finite element electromagnetics," IEEE Trans. Magn., vol. 30(5), pp. 3523-3526, 1994.

[3] N. A. Golias and T. D. Tsiboukis, "Adaptive methods in computational magnetics," Int. J. Num. Mod.: E.N.D.F., vol. 9, pp. 71-80, 1996.

[4] S. McFee and D. Giannacopoulos, "Optimal discretization based refinement criteria for finite element adaption," IEEE Trans. Magn., vol. 32(3), pp. 1357-1360, 1996.

[5] I. Babuska and R. Rodriguez, "The problem of the selection of an a posteriori error indicator based on smoothening techniques," Int. J. Num. Meth. Eng., vol. 36, pp. 539-567, 1993.

[6] D. Giannacopoulos and S. McFee, "An experimental study of superconvergence phenomena in finite element magnetics," IEEE Trans. Magn., vol. 33(5), pp. 4137-4139, 1997. 